\title{
Ethische Standards für die Beratung vor pränataler Diagnostik?
}

\author{
E. Bühler
}

Richtlinien oder Standards sind heute in Mode. Das mag damit zusammenhängen, dass Gesetze, Vorschriften, Gebote und Verbote unbeliebt sind, das Bedürfnis nach Sicherheit aber eben doch vorhanden ist. Mit der Zunahme technischer Möglichkeiten wächst die Unsicherheit in bezug auf deren Wünschbarkeit; man möchte wenigstens wissen, unter welchen Bedingungen sie angewendet werden sollen. Ethik in der Medizin ist heute mehr denn je gefragt. Auch das hängt mit dem schnellen Fortschritt in der Forschung und deren Anwendungsmöglichkeiten in Diagnostik, Therapie und Prophylaxe zusammen.

Solche Überlegungen haben den «Verein für unabhängige Beratung und Information über pränatale Diagnostik» (Verein UBI) veranlasst, sich Gedanken über ethische Standards in der Beratung vor pränataler Diagnostik zu machen.

Der Verein UBI entstand aus einer Arbeitsgruppe von Frauen, die 1993 im Auftrag des Ökumenischen Forums christlicher Frauen ein Papier über pränatale Diagnostik verfasste. Aus der Beschäftigung mit diesem Thema erwuchs bei einigen der Frauen der Wunsch, sich auch praktisch in der Hilfe für Menschen, die mit diesem Problem konfrontiert sind, zu engagieren. Die individuelle Hilfe sollte absolut im Vordergrund stehen; wir wollten uns an keine weltanschauliche Doktrin binden. So entstand 1995 der Verein UBI, bestehend aus einer Genetikerin, zwei Hebammen, zwei Naturwissenschaftlerinnen, einer Gymnasiallehrerin und einer Mutter eines Kindes mit Down Syndrom. Präsidentin wurde die Inhaberin des Lehrstuhls für Sozial- und Präventivmedizin der Universität Basel.

Die Abkürzung UBI entstand erst 1999, weil sich der ganz ausgesprochene oder ausgeschriebene Name als zu lang und kompliziert erwies. Die drei Buchstaben beinhalten das, was uns am wichtigsten ist: Die Unnabhängigkeit der Beratung und eine objektive Information, die nicht nur dem neuesten Stand des Wissens entspricht, sondern auch der individuellen Situation Rechnung trägt. Unterbrechung der Schwangerschaft, Behinderung des Kindes, Individualität der Entscheidung könnten drei andere Worte mit den Anfangsbuchstaben U, B, I sein, die die Probleme benennen, mit denen sich die Ratsuchende aus- einandersetzen muss, wenn sie vor einer pränatalen Diagnostik steht. Und wenn man philosophisch veranlagt ist, kann man aus dem Wort ubi? (dem lateinischen wo?) auch die Frage heraushören, wohin uns wohl die Entwicklung neuer diagnostischer Möglichkeiten während der Schwangerschaft führen wird.

Richtlinien der Schweizerischen Akademie der Medizinischen Wissenschaften (SAMW) über genetische Untersuchungen am Menschen gibt es schon seit bald 10 Jahren (die jetzige Präsidentin des Vereins UBI war übrigens Mitglied der für die Ausarbeitung der Richtlinien tätigen Subkommission), und ein Bundesgesetz ist im Entstehen begriffen. Für die Anwendung genetischer Tests werden also Gesetze formuliert und Richtlinien herausgegeben, nicht aber für die Beratung der Einzelperson vor und nach solchen Untersuchungen. Dass Beratungsangebote geschaffen werden müssen, wird zwar betont, wie diese aber aussehen und wie sie agieren sollen, steht nirgends. In der Schweiz liegt bis heute kein detailliertes Beratungskonzept zu pränatalen Untersuchungen vor, von ethischen Richtlinien ganz zu schweigen.

Im Verlaufe unserer Beratungstätigkeit ist uns klar geworden, dass es auch für die Beratung selbst Richtlinien braucht, nicht nur für die Durchführung genetischer Tests. Aus dieser Überlegung entstanden Postulate für Standards in der Beratung für vorgeburtliche Untersuchungen. Diese sollen im folgenden dargestellt werden:

1. Unvoreingenommenheit: Eine Beraterin, die absolute Abtreibungsgegnerin ist, wird nicht unvoreingenommen über Für und Wider der pränatalen Diagnostik reden können. Ein Berater, der in einem Behinderten nur eine Last für Staat, Gesellschaft und Familie sieht, wird kaum unabhängig von dieser Ansicht beraten können.

2. Verantwortungsbewusstsein: Ein ausgeprägtes Verantwortungsbewusstsein ist zweifelsohne absolute Voraussetzung für eine ethisch hochstehende Beratung. Als Berater sind wir nicht nur mitverantwortlich für die Entscheidung, die eine Ratsuchende trifft, wir wirken auch als Meinungsbildner für die Ein- 
stellung der Gesellschaft zu Behinderung, Lebensqualität, Gesundheit und Krankheit. Ein fundiertes Wissen und eine gute sachliche Information gehören mit zu der Verantwortung von Beraterinnen und Beratern.

3. Empathie, verbunden mit Einfühlungsvermögen: sind weitere Postulate einer Ethik für Beratende. In den oft schwierigen Entscheidungen von Für und Wider pränatal diagnostischer Massnahmen oder von Konsequenzen pränataler Untersuchungen fühlen Frauen sich von Frauen oft besser verstanden als von Männern. Empathie wächst auf dem Boden des Einfühlungsvermögens, und verständlicherweise kann eine Frau sich besser in eine Schwangere einfühlen als ein Mann.

4. Zeitaufwand: Es mag befremden, genügenden Zeitaufwand als Postulat einer ethisch verantworteten Beratung $\mathrm{zu}$ nennen. Die Erfahrung lehrt aber, dass Gespräche unter Zeitdruck ein Hindernis für eine freie, selbstverantwortete Entscheidung sind. Jeder Beratung sollte das Angebot von Fortsetzungsgesprächen folgen.

5. Respekt und Solidarität: Respekt vor der Freiheit der ratsuchenden Person führt zur Solidarität mit ihr. Dieser Respekt verlangt vom Beratenden grösstmögliche Zurückhaltung im Offenlegen seiner persönlichen Meinung. Sobald eine Entscheidung gefallen ist, ist nur noch Solidarität mit der Klientin gefragt.

6. Vernunft: Dieses letzte Postulat soll weniger als ethischer Standard verstanden werden, sondern eher ein praktischer Hinweis für die Beratung sein. Da es nie gelingen wird, allen Risiken einer Schwangerschaft zu begegnen, allen Gefahren für das Kind auszuweichen und alle Zwischenfälle zu vermeiden, sollte man bemüht sein, im Gespräch mit der ratsuchenden Person aus allem Machbaren das für ihren Fall Wünschbare herauszuarbeiten. Voraussetzung dafür ist - neben der eigenen Einsicht, dass nicht alles Machbare auch wünschenswert ist - das Geschick oder die Gabe zu beurteilen, was im Einzelfall das Vernünftigste ist.

Aus sechs Jahren Beratungspraxis sollen im folgenden einige Erfahrungen wiedergegeben werden. Die telefonischen oder schriftlichen Anfragen lassen sich grosso modo in zwei Kategorien unterteilen: Auf der einen Seite reine Informationsübermittlung (Vor- und Nachteile verschiedener Methoden, Risiko der Eingriffe, Aussage- kraft von Resultaten und Befunden), auf der anderen Entscheidungshilfe und eigentliche Beratung (Für und Wider bzw. Wahl pränatal diagnostischer Massnahmen, Vorgehen nach zwiespältigen, fraglichen oder eindeutig pathologischen Befunden). Oft umfasst ein Gespräch beide Kategorien, manchmal nur eine davon:

1. Die erste Kategorie hat in den sechs Jahren des Bestehens des Vereins UBI eine auffallende Wandlung erfahren. Die Frauen sind jetzt besser informiert als früher, was die Möglichkeiten der pränatalen Diagnostik betrifft. Verständnisfragen treten noch auf bei der Aussagekraft von Risikoangaben (Wahrscheinlichkeiten), bei den Ausdrücken «positiver» bzw. «negativer» Befund und bei der Bedeutung eines normalen Chromosomenbefundes für die Gesundheit bzw. Normalität des Kindes. Es ist immer noch ein weitverbreitetes Phänomen, dass Frauen nach Erhalt eines normalen Chromosomenbefundes sich in der falschen Sicherheit wiegen, ihr Kind würde nun garantiert gesund zur Welt kommen. Man könnte es so ausdrücken: Die Frauen sind heutzutage gut informiert über die Möglichkeiten, weniger gut über die Grenzen der pränatalen Diagnostik.

2. Die zweite Kategorie von Anfragen wird wahrscheinlich immer gleich aktuell sein. Da jede Ratsuchende sich in einer anderen persönlichen Situation befindet, sehen wir die primäre Aufgabe der Beraterin darin, herauszuspüren, wie die Frau selber über ihre Situation denkt, was sie fühlt in bezug auf ihre Schwangerschaft, wie sie zu Fragen wie Sicherheit und Risikobereitschaft steht, was für ein Menschenbild sie und ihr Partner haben, was für eine Weltanschauung sie vertreten. All das wird meistens nicht direkt gefragt, sondern wir versuchen, es durch das Gespräch zu erspüren. Sodann bestärken wir die Ratsuchende in der von ihr angedeuteten (selten klar ausgesprochenen) Präferenz oder in der von ihr anvisierten Entscheidung. Es ist unsere Erfahrung, dass Frauen leichter $\mathrm{zu}$ einer für sie stimmigen Entscheidung kommen, wenn sie sich ernst genommen fühlen und nicht etwa für naiv und unaufgeklärt oder umgekehrt überängstlich und perfektionistisch gehalten werden. Ein bestimmtes Risiko kann von einem Menschen als hoch und inakzeptabel angesehen werden, während der gleiche Befund für ein anderes Paar kein Grund zum Schwangerschaftsabbruch ist. 
Die Frauen des Beratungsteams im Verein UBI sehen sich nicht als Konkurrenz der Ärzte, auch nicht als Lehrpersonen, Richterinnen oder moralische Instanz, sondern als Informanten, Zuhörerinnen, Beraterinnen und Helferinnen (meist) schwangerer Frauen und deren Partner in schwierigen persönlichen Situationen.

Von seiten der Ärzteschaft haben wir eher passiven als aktiven Widerstand erfahren. Wir wünschten uns mehr positives Echo und ideelle Unterstützung. Dass unser Angebot gratis ist und alle Mitarbeiterinnen ehrenamtlich arbeiten, schadet vielleicht unserem Ansehen in den Augen mancher Ärzte, aber auch mancher ähnlicher Organisationen und eventuell auch potentieller Benutzerinnen. Es ist auch nicht einfach, geeignete Mitarbeiterinnen zu rekrutieren. In der jetzigen Zusammensetzung des Teams hat die Genetikerin das Präsidium inne, eine Naturwissenschaftlerin mit persönlicher Erfahrung pränataler Diagnostik das Vizepräsidium. Zwei Hebammen, eine Krankenschwester und eine weitere Frau mit persönlichen Erfahrungen pränataler Diagnostik sind Mitglieder des Vorstands und des Beratungsteams. Alle diese Frauen sind auch Mütter, z.T. schon Grossmütter. Für ganz spezielle Fragestellungen steht gegebenenfalls ein weiterer Kreis von Fachfrauen zur Verfügung: Frauen- und Kinderärztinnen, Theologinnen, eine Psychiaterin, Mütter- und Väterberaterinnen, Sozialarbeiterinnen und eine Advokatin. Eine ebenfalls ehrenamtlich arbeitende Kassierin nimmt sich der spärlichen Finanzen des Vereins UBI an. Der Kreis zahlender Mitglieder des Vereins beläuft sich seit Jahren auf 25 bis 30 .
Jüngere berufstätige Frauen mit kleinen Kindern bleiben oft nur kurze Zeit in unserem Beratungsteam. Wir sind darum immer dankbar um Interessentinnen aus dem Raume Basel. Wünschenswert ist natürlich eine irgendwie nicht ganz «blande Anamnese» in bezug auf die Problematik von Schwangerschaft, Geburt, pränataler Diagnostik, sei es von der Ausbildung her, sei es von persönlichen Lebenserfahrungen oder beruflicher Tätigkeit. Junge Ärztinnen, die vielleicht gerade eine «Babypause» machen oder nur Teilzeit arbeiten, Biologielehrerinnen, Krankenschwestern, Hebammen in ähnlicher Situation, aber auch Frauen, die Erfahrung mit behinderten Kindern haben, sind immer willkommen. Die spezifische Ausbildung erfolgt im Verein UBI durch interne Fortbildung in Form von Merkblättern, Teamsitzungen mit Erfahrungsaustausch und gelegentlichen Fortbildungsveranstaltungen.

Selbstverständlich sind wir auch für Mitglieder aus anderen Teilen der Schweiz oder dem Ausland dankbar, denn die Infrastruktur, so klein sie ist, braucht doch ein minimales finanzielles Polster. Die Mitgliedschaft steht jeder interessierten Einzelperson (Jahresbeitrag Fr. 30.-) oder jedem Kollektiv (Jahresbeitrag Fr. 200.-) offen.

Momentan ist eine «Broschüre für werdende Eltern» über Fragen der pränatalen Diagnostik im Entstehen. Sie wird voraussichtlich noch in diesem Jahr erhältlich sein. Wir werden den Preis so gering wie möglich halten. Interessenten und Interessentinnen können sich an die Autorin dieses Beitrags, an die Vereins- oder die E-MailAdresse wenden: Verein für unabhängige Beratung und Information über pränatale Diagnostik (Verein UBI), Mittlere Strasse 35, 4056 Basel, E-Mail: consultant@netlimit.com. 